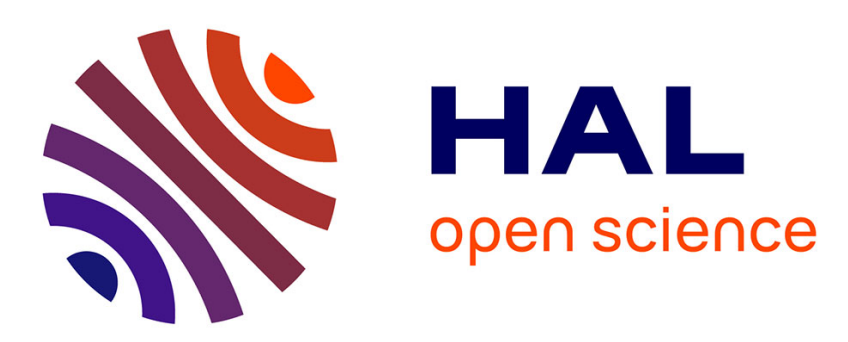

\title{
Transmission des informations médicales et projet d'enfant : le conseil génétique dans le contexte du rétinoblastome et du cancer du sein et des ovaires dit héréditaire
}

Catherine Dekeuwer

\section{To cite this version:}

Catherine Dekeuwer. Transmission des informations médicales et projet d'enfant : le conseil génétique dans le contexte du rétinoblastome et du cancer du sein et des ovaires dit héréditaire. Ethique \& santé, 2013, 10 (1), pp.34-42. 10.1016/j.etiqe.2012.11.009 . hal-02950186

\section{HAL Id: hal-02950186 \\ https://univ-lyon3.hal.science/hal-02950186}

Submitted on 27 Sep 2020

HAL is a multi-disciplinary open access archive for the deposit and dissemination of scientific research documents, whether they are published or not. The documents may come from teaching and research institutions in France or abroad, or from public or private research centers.
L'archive ouverte pluridisciplinaire HAL, est destinée au dépôt et à la diffusion de documents scientifiques de niveau recherche, publiés ou non, émanant des établissements d'enseignement et de recherche français ou étrangers, des laboratoires publics ou privés. 


\section{Elsevier Editorial System(tm) for \&\#xC9; thique et Sant\&\#xE9;}

Manuscript Draft

\section{Manuscript Number:}

Title: Transmission des informations médicales et projet d'enfant : le conseil génétique dans le contexte du rétinoblastome et du cancer du sein et des ovaires dit héréditaire Medical information and the plan to have a child: genetic counseling in the context of retinoblastoma and hereditary breast and ovarian cancer

Article Type: Article original

Keywords: Cancers héréditaires; Projet d'enfant; DPN et DPI; Conseil génétique; Autonomie reproductive

Hereditary Cancers / Plan to have a child / PND and PGD / Genetic counseling / Reproductive autonomy

Corresponding Author: Dr CATHERINE DEKEUWER, Ph.D

Corresponding Author's Institution: Université Lyon 3 Jean Moulin

First Author: CATHERINE DEKEUWER, Ph.D

Order of Authors: CATHERINE DEKEUWER, Ph.D

Abstract: Les consultations de conseil génétique visent à donner des informations sur les buts et la nature des tests génétiques ainsi que sur les implications de leurs résultats, notamment sur les choix procréatifs. Elles s'adressent donc aux personnes qui envisagent d'avoir un enfant alors qu'elles risquent de transmettre une anomalie génétique associée à une maladie grave. Selon la pathologie, le recours au diagnostic anténatal (DPN et DPI) peut y être discuté. Or, en matière de conseil génétique, le principe de non-directivité guide les recommandations de bonne pratique : il s'agit d'aider le couple concerné à faire ses propres choix, de manière libre et éclairée. En ce sens, ces consultations favorisent l'autonomie reproductive des couples et protègent leur liberté de décision. Cependant, ces consultations ne sont pas le seul lieu où les questions relatives au projet d'enfant sont posées ; à quel moment de leur parcours médical les personnes qui risquent de transmettre une anomalie génétique à leurs enfants bénéficient-elles d'informations pertinentes pour leurs décisions procréatives ? De plus, le contenu des informations ainsi que la manière dont elles sont transmises ont un impact sur les réflexions et les décisions procréatives. Les résultats d'une enquête menée auprès de personnes qui connaissaient leur risque d'avoir transmis ou de transmettre à leurs enfants une mutation génétique associée à un risque accru de cancer permettent d'identifier plusieurs entraves à leur autonomie reproductive.

Genetic counseling aims to provide information about the goals and the nature of genetic tests and about the implications of test results, notably for reproductive choices. Consequently, it is addressed to persons who wish to have children but are at risk of transmitting to them a genetic anomaly associated with severe disease. Depending on the pathology, prenatal diagnosis (PND) or preimplantation genetic diagnosis (PGD) are options that can be discussed. The principle of non-directive genetic counseling underlies recommendations for good practice: the aim of genetic counseling is to help the couple to make its own free and informed decision. In this sense, it promotes the reproductive autonomy of the 
couples and protects their freedom of choice. However, these consultations are not the only place where questions regarding the prospect of having children are raised: at what moments in their medical itinerary do persons at risk of transmitting a genetic mutation to their offspring receive information that is relevant to reproductive decision-making? Moreover, the content of the information as well as the way in which they are conveyed have an impact on reproductive reflections and decisions. The results of a study based on interviews with persons who know they are at risk of transmitting to their offspring a genetic mutation associated with an increased risk of cancer reveal several impediments to reproductive autonomy. 
Transmission des informations médicales et projet d'enfant: le conseil génétique dans le contexte du rétinoblastome et du cancer du sein et des ovaires dit héréditaire

Medical information and the plan to have a child: genetic counseling in the context of retinoblastoma and hereditary breast and ovarian cancer

Catherine DEKEUWER

Maître de conférences, Faculté de philosophie, Université Lyon 3 Jean Moulin

Chercheur associée au CERSES (CNRS-Université Paris-Descartes)

1, rue de l'université 69007 Lyon (France)

catherine.dekeuwer@univ-lyon3.fr

$+33661448011$

Soutiens financiers :

Agence de la Biomédecine

Fondation de France

Aucun conflit d'intérêt 
Transmission des informations médicales et projet d'enfant : le conseil génétique dans le contexte

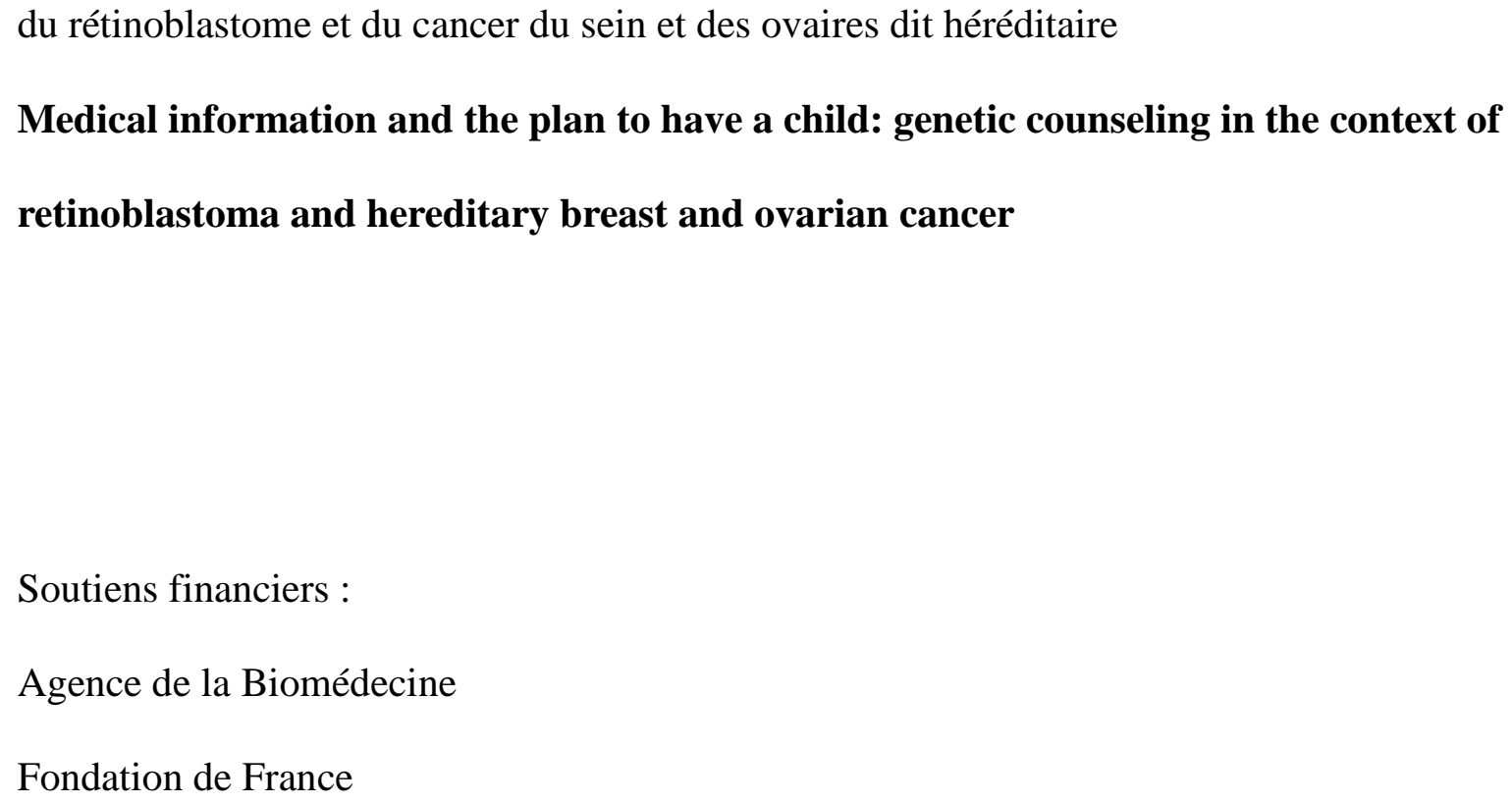




\section{Résumé :}

Les consultations de conseil génétique visent à donner des informations sur les buts et la nature des tests génétiques ainsi que sur les implications de leurs résultats, notamment sur les choix procréatifs. Elles s'adressent donc aux personnes qui envisagent d'avoir un enfant alors qu'elles risquent de transmettre une anomalie génétique associée à une maladie grave. Selon la pathologie, le recours au diagnostic anténatal (DPN et DPI) peut y être discuté. Or, en matière de conseil génétique, le principe de non-directivité guide les recommandations de bonne pratique : il s'agit d'aider le couple concerné à faire ses propres choix, de manière libre et éclairée. En ce sens, ces consultations favorisent l'autonomie reproductive des couples et protègent leur liberté de décision. Cependant, ces consultations ne sont pas le seul lieu où les questions relatives au projet d'enfant sont posées ; à quel moment de leur parcours médical les personnes qui risquent de transmettre une anomalie génétique à leurs enfants bénéficient-elles d'informations pertinentes pour leurs décisions procréatives ? De plus, le contenu des informations ainsi que la manière dont elles sont transmises ont un impact sur les réflexions et les décisions procréatives. Les résultats d'une enquête menée auprès de personnes qui connaissaient leur risque d'avoir transmis ou de transmettre à leurs enfants une mutation génétique associée à un risque accru de cancer permettent d'identifier plusieurs entraves à leur autonomie reproductive.

\section{Mots clés}

Cancers héréditaires ; Projet d'enfant ; DPN et DPI ; Conseil génétique ; Autonomie reproductive 


\begin{abstract}
Genetic counseling aims to provide information about the goals and the nature of genetic tests and about the implications of test results, notably for reproductive choices. Consequently, it is addressed to persons who wish to have children but are at risk of transmitting to them a genetic anomaly associated with severe disease. Depending on the pathology, prenatal diagnosis (PND) or preimplantation genetic diagnosis (PGD) are options that can be discussed. The principle of nondirective genetic counseling underlies recommendations for good practice: the aim of genetic counseling is to help the couple to make its own free and informed decision. In this sense, it promotes the reproductive autonomy of the couples and protects their freedom of choice. However, these consultations are not the only place where questions regarding the prospect of having children are raised: at what moments in their medical itinerary do persons at risk of transmitting a genetic mutation to their offspring receive information that is relevant to reproductive decision-making? Moreover, the content of the information as well as the way in which they are conveyed have an impact on reproductive reflections and decisions. The results of a study based on interviews with persons who know they are at risk of transmitting to their offspring a genetic mutation associated with an increased risk of cancer reveal several impediments to reproductive autonomy.
\end{abstract}

\author{
Key words : \\ Hereditary Cancers ; Plan to have a child ; PND and PGD ; Genetic counseling ; Reproductive \\ autonomy
}




\section{Introduction}

Pour les personnes qui risquent de transmettre une anomalie génétique associée à une maladie grave, prendre la décision d'avoir un enfant est parfois difficile. Certains couples choisissent de prendre le risque de transmettre l'anomalie génétique, mais d'autres renoncent à leur projet parental ou s'orientent vers une démarche d'adoption. D'autres encore souhaiteraient bénéficier d'une aide médicale, et notamment recourir au diagnostic anténatal [1-4]. Le diagnostic prénatal (DPN) permet de savoir si l'embryon ou le fœetus est porteur de l'anomalie génétique ; les parents peuvent alors choisir de ne pas poursuivre la grossesse. Le diagnostic préimplantatoire (DPI) permet d'éviter la transmission de l'anomalie génétique puisque seuls des embryons non porteurs, obtenus par fécondation in vitro (FIV), seront transférés dans l'utérus maternel. Même s'il existe encore peu d'études sur la question, elles suggèrent que la réflexion des couples confrontés à cette situation est complexe [3-7].

Ces réflexions sur le projet d'enfant s'effectuent dans le cadre d'une prise en charge médicale. Des consultations dites de « conseil génétique » visent à donner des informations sur les buts et la nature des tests génétiques ainsi que sur les implications de leurs résultats, notamment en ce qui concerne les choix procréatifs [8]. Depuis les années 1950, le principe de non-directivité guide les recommandations de bonnes pratiques : il s'agit d'aider la personne concernée à faire ses propres choix, de manière libre et éclairée [8-11]. En ce sens, ces consultations favorisent l'autonomie reproductive des couples et protègent leur liberté de décision. Cette liberté de décision est encadrée : en France, les couples qui souhaiteraient recourir au diagnostic anténatal doivent obtenir une attestation de gravité auprès d'un Centre pluridisciplinaire de diagnostic prénatal (CPDPN). Ces instances hospitalières sont chargées de « contrôler le bien-fondé de la demande des couples » [12] et sont garantes du respect de la loi. En France, le recours au DPN ou au DPI est autorisé uniquement lorsque l'embryon ou le fœtus a un risque élevé d'être atteint d'une maladie «d'une particulière gravité et incurable au moment du diagnostic » [13]. Selon les maladies, 
l'interprétation des termes de la loi pose plus ou moins de difficultés aux médecins.

Cependant, les questions relatives au projet d'enfant ne sont pas abordées uniquement lors des consultations de conseil génétique et il est utile de savoir à quel moment de leur parcours médical les personnes qui risquent de transmettre une anomalie génétique à leurs enfants bénéficient d'informations pertinentes pour leurs décisions procréatives. De plus, le contenu des informations ainsi que la manière dont elles sont transmises ont un impact sur les réflexions et les décisions procréatives [8-12,14,15]. Nous avons mené une enquête auprès de personnes qui connaissaient leur risque d'avoir transmis ou de transmettre à leurs enfants une anomalie génétique associée à un risque accru de cancer afin de mieux connaître leurs expériences, leurs questionnements et leurs raisonnements à propos du projet d'enfant. Les résultats de cette étude permettent d'identifier plusieurs entraves à leur autonomie reproductive.

\section{Description de l'enquête}

Les résultats présentés ici sont issus de l'analyse d'entretiens exploratoires semi-directifs menés avec des personnes qui se savaient porteuses d'une mutation génétique associée à un risque accru de cancer (voir le tableau 1). Elles étaient confrontées à des maladies de gravité différentes [16] et pour lesquelles le recours au diagnostic anténatal est considéré comme plus ou moins acceptable par les médecins.

Nous avons rencontré dix personnes porteuses de mutations du gène $R B 1$ liées au développement de tumeurs dans la rétine. Le rétinoblastome est une maladie peu fréquente qui atteint les enfants très jeunes, parfois même avant la naissance. Des traitements permettent de lutter contre ces tumeurs et selon la précocité de la prise en charge et leur localisation, les séquelles sont plus ou moins importantes. Le risque d'atteinte est variable : il existe des familles dans lesquelles tous les porteurs de l'anomalie génétique développent des tumeurs, alors que dans d'autres familles certains porteurs n'en développent pas. Même si cette maladie n'est pas à strictement parler incurable, certains couples ont reçu les attestations de gravité leur permettant de recourir au DPN et 
au DPI $[17,18]$.

Vingt et un entretiens concernent les cancers du sein et des ovaires dits héréditaires. Le risque de développer des tumeurs varie selon le sexe, les familles et les mutations ${ }^{1}$. Dans certaines familles, le cancer peut toucher des femmes avant leur trentième anniversaire. En dépit des mesures de surveillances et de prévention (ovariectomie et mammectomie prophylactiques), certaines personnes estiment que la maladie est si grave et les mesures de prévention si radicales qu'elles préféreraient ne pas transmettre une mutation des gènes $B R C A 1$ ou $B R C A 2$ à leurs enfants. Alors que les attestations de gravité permettant de recourir au DPI n'étaient jamais données dans ce contexte, pour la première fois en France un couple en a obtenu une il y a quelques mois.

A titre complémentaire, nous utilisons des données issues de deux entretiens relatifs à la polypose adénomateuse familiale (PAF). En l'absence de chirurgie prophylactique, le risque de cancer du côlon est estimé à $100 \%$ à 40 ans. La sévérité de la maladie varie considérablement, notamment en raison du développement d'autres tumeurs particulièrement difficiles à traiter. Une surveillance des enfants porteurs d'une mutation du gène $A P C$ est préconisée dès l'âge de 10-12 ans, suivie d'une colectomie quelques années plus tard. En 2010, quatre demandes de DPI ont été examinées en France et deux ont été acceptées [18].

Nous avons recruté ces personnes dans deux centres français spécialisés en génétique où elles étaient invitées à procéder à un entretien de manière volontaire et anonyme ${ }^{2}$. Lorsqu'une personne et son conjoint le souhaitaient, une partie de l'entretien était effectuée avec le couple. Nous avons ensuite analysé ces entretiens selon une méthode qualitative classique, consistant à identifier des thèmes, des idées ou des raisonnements récurrents et à les comparer. L'approche inductive du matériel a permis également à des thèmes non attendus d'émerger.

Nos résultats s'appuient également sur des entretiens menés avec des médecins qui travaillent dans ces centres. Les deux chefs de service, quatre médecins qui assurent des

\footnotetext{
1 Les femmes ont un risque cumulé de cancer du sein estimé entre 50 et $70 \%$ à 70 ans et un risque de cancer des ovaires estimé à 10-40\% à 70 ans [16]. Les hommes ont un risque cumulé de développer un cancer du sein estimé à $7 \%$ ainsi qu'un risque accru de cancer de la prostate [19].

2 Notre enquête a été approuvée par la CNIL.
} 
consultations de génétique ainsi qu'un psychiatre et deux psychologues ont accepté de nous

rencontrer. Dans un centre, nous avons aussi rencontré deux médecins qui interviennent dans le cadre d'un parcours de surveillance dédié aux femmes qui n'ont pas été malades mais portent une mutation BRCA1/2. Ces entretiens avaient pour fin de mieux comprendre l'organisation du parcours de soin et les modalités de prise en charge relatives à chaque maladie. Nous demandions également à chaque médecin si la question de la reproduction était abordée en consultation, et comment.

\section{1) En amont des consultations de génétique : le projet d'enfant ?}

Un des intérêts de la démarche de recherche génétique est de préciser qui, dans une famille, est porteur de l'anomalie génétique. Si les membres de la famille choisissent de faire un test génétique, les non porteurs peuvent alors être rassurés, tandis qu'une prise en charge médicale commence pour les porteurs. Mais s'engager dans cette démarche implique d'aborder la question de la transmission: un point commun des consultations de génétique est de tracer un arbre généalogique. Cette question est posée au passé, mais elle peut aussi concerner le futur : quelles sont, et quelle place ont, dans ce contexte, les questions qui concernent le fait d'avoir des enfants ?

\subsection{Le cas du rétinoblastome}

Les questions relatives au fait d'avoir des enfants sont parfois initiatrices de la démarche conduisant à connaître son statut génétique. Parmi les personnes porteuses d'une mutation du gène $R B 1$ que nous avons rencontrées, six ont consulté en génétique alors qu'elles souhaitaient avoir leur premier enfant. Toutes ces personnes savaient que la maladie, dont certains avaient souffert dans l'enfance, était génétique. Parfois, des recherches génétiques avaient déjà été effectuées par des apparentés qui ensuite les avaient informés. Certains se souviennent aussi que lors de consultations relatives à leur surveillance médicale, les médecins avaient insisté sur l'importance de cette consultation de conseil génétique. Une jeune femme raconte par exemple qu'à l'adolescence, un 
médecin lui a dit : «si un jour tu veux des enfants, il faut absolument qu'on se voit, il faut qu'on en

discute parce que tu peux avoir des enfants qui ont la même maladie que toi ».

Parmi ces personnes, trois avaient choisi de consulter pour connaître leur risque de transmettre leur anomalie génétique à leur enfant. Une femme explique que pour la construction de son couple, et avant même que le projet d'avoir un enfant ne se précise, « on avait besoin de savoir si c'était possible [d'avoir un enfant] et dans quelles conditions ». Deux personnes avaient réalisé le test génétique vers l'âge de vingt ans et étaient retournées en consultation de génétique avec leur partenaire au moment de «mettre en route » leur premier enfant. Pour l'un d'entre eux, devenu aveugle à cause du rétinoblastome, il s'agissait alors de trouver une solution pour ne pas risquer de transmettre l'anomalie génétique : sa compagne «voulait un enfant qui ne soit pas handicapé ». Pour les autres, il s'agissait de connaître les risques de transmission et les possibilités de prise en charge médicale. Enfin, une femme avait entendu parler du DPI lors d'une conférence grand publique ; elle a choisi de consulter pour discuter spécifiquement de cette option.

C'est la maladie d'un enfant dans la famille qui a conduit les quatre autres personnes à réaliser un test génétique. Deux personnes n'avaient pas été malades dans l'enfance : c'est la maladie d'un neveu dans un cas et celle du premier né dans l'autre cas qui les ont conduit en consultation de génétique. Les deux autres personnes avaient été malades dans l'enfance, mais elles ne savaient pas que leur maladie était liée à une mutation génétique. Dans ce contexte, un homme raconte avoir posé des questions à son ophtalmologue et au gynécologue de sa compagne au moment d'avoir un enfant : il redoutait que sa maladie soit héréditaire. Ces deux médecins l'ont rassuré : «il n'y a aucune chance que vous transmettiez la maladie ». Pourtant, sa fille est née avec des tumeurs dans un œil. Pour leur second enfant, le couple a déposé une demande de DPI : ils souhaitent lui éviter cette maladie et tous les problèmes qui y sont associés.

La prise en charge médicale du rétinoblastome donne donc une place aux questions qui concernent le fait d'avoir des enfants : c'est parce que des apparentés ont eux-mêmes bénéficié de consultations de génétique ou parce que d'autres médecins ont souligné leur importance que les 
personnes que nous avons rencontrées ont pu poser les questions qui les préoccupaient : quel est le

risque de transmettre l'anomalie génétique ? Quelles en sont les conséquences ? Quels sont les moyens de l'éviter? En amont des consultations de génétique, c'est alors l'importance de la formation des médecins « de ville» qu'il convient de souligner. Lorsqu'elles s'exprimaient sur cette question, les personnes que nous avons rencontrées expliquaient en effet que les pédiatres, ophtalmologues, gynécologues et médecins généralistes devraient être capables non seulement de diagnostiquer la maladie mais aussi d'orienter en consultation de génétique. Or, ce n'est pas toujours le cas. Faute d'information, certaines personnes n'ont pas pu prendre les décisions procréatives qu'elles estiment pourtant être les meilleures. D'autres au contraire parlaient de la chance qu'elles avaient eu de pouvoir bénéficier d'un suivi médical leur permettant de prendre la meilleure décision possible. L'exercice de l'autonomie reproductive dépend donc des modalités d'accès à l'information génétique. Son importance est soulignée dans d'autres contextes : nous avons en effet rencontré des porteurs de mutations des gènes $R B 1, A P C, B R C A 1$ et $B R C A 2$ engagés auprès d'associations de malades ou qui avaient témoigné dans différents médias pour mieux faire connaître la démarche de génétique et son intérêt pour les décisions procréatives.

\subsection{Le cas du cancer du sein et des ovaires}

Certaines femmes que nous avons rencontrées avaient été adressées en consultation de génétique pendant ou juste après un cancer du sein ou des ovaires, par exemple par leur chirurgien ou leur radiothérapeute. Les autres personnes avaient été informées de la présence d'une mutation BRCA1/2 dans la famille par un apparenté plus ou moins proche. Toutes les femmes, ainsi que l'un des deux hommes, étaient préoccupés par leur prise en charge médicale et celle de leurs apparentés. Pour celles qui avaient développé un cancer, la motivation principale pour faire le test génétique était la protection de leurs apparentées : grâce à cette information génétique, leur filles et petitesfilles, leurs sœurs, nièces, etc. pourraient bénéficier de mesures de surveillances et de prévention efficaces. Qu'elles aient ou non été malades, ces femmes cherchaient aussi à être rassurées : si leur 
test s'avérait négatif, elles seraient soulagées de ne pas avoir transmis ou de ne pas pouvoir

transmettre l'anomalie génétique à leurs enfants.

Des questions sur la transmission sont donc au cœur de la démarche de recherche génétique. Mais pour les femmes qui ont le projet d'avoir un enfant, les aborder peut être très difficile. Pour certaines, les bénéfices d'une prise en charge précoce prennent le pas sur ces préoccupations. A l'inverse, une femme explique qu'elle a attendu d'avoir tous ses enfants avant de se rendre en consultation de génétique. Elle savait bien que sa sœur aînée, décédée des suites d'un cancer du sein, avait fait un test génétique. Elle connaissait aussi l'importance de cette information pour sa prise en charge. Pourtant, prendre le risque de transmettre la mutation en connaissance de cause était trop difficile. Les porteurs choisissent aussi parfois de ne pas avoir d'enfants. Ainsi, selon une étude effectuée auprès de personnes qui envisageaient de réaliser un test génétique [20], 36\% rapportaient qu'ils auraient des enfants quel que soit le résultat, tandis que $12 \%$ pensaient qu'ils n'auraient pas d'enfants s'ils étaient porteurs ${ }^{3}$.

Notre enquête suggère qu'à l'inverse de ce qui se passe dans le contexte du rétinoblastome, les préoccupations relatives au risque de transmettre une anomalie génétique à ses enfants ne sont que rarement à l'origine ou au premier plan des consultations de génétique concernant les cancers du sein et des ovaires. Une seule personne a souhaité réaliser le test génétique dans ce but précis. Cet homme a eu deux enfants nés après deux DPI réalisés à l'étranger : il était exclu, de son point de vue, de prendre le risque de transmettre l'anomalie génétique responsable des décès précoces de sa mère puis de sa sœur. L'une des raisons de cette différence tient au parcours médical des personnes que nous avons rencontrées. Dans le contexte d'une histoire familiale de cancers du sein et des ovaires, la consultation de génétique est en effet conseillée pour bénéficier d'une meilleure prise en charge médicale. Mais supposons que le diagnostic anténatal soit considéré comme une solution pour les parents qui ne souhaiteraient pas prendre le risque de transmettre une mutation corrélée à

3 En plus de ces $12 \%$ qui n'auraient pas d'enfants, 55\% considéreraient le DPN, $48 \%$ le DPI et $30 \%$ l'adoption. 
une forme familiale particulièrement sévère de cancer du sein ou des ovaires : les questions

relatives à la procréation deviendraient sans doute, comme pour le rétinoblastome, une motivation pour consulter un généticien.

\section{2) La place des réflexions sur la procréation lorsque le recours au diagnostic anténatal} est controversé

D'après notre enquête, la place accordée aux questions procréatives en consultation de génétique dépend des solutions que les médecins estiment pouvoir apporter aux couples. Ainsi, dans le cas du rétinoblastome, les médecins que nous avons rencontrés expliquent que les questions relatives à la procréation sont abordées pendant la consultation d'information concernant l'intérêt de réaliser un test génétique ou pendant la consultation de rendu de résultat. Ils informent du risque de transmission de la mutation génétique et de ses conséquences pour l'enfant. Ils présentent aussi trois options au couple : surveiller l'enfant dès sa naissance, procéder à une interruption médicale de grossesse (IMG) après un DPN ou recourir au DPI. Ces options sont discutées au cas par cas. Les médecins informent également leurs patients des risques de transmission des mutations BRCA1/2, mais n'engagent pas plus avant de discussion sur la procréation : c'est uniquement si le patient pose une question ou met en avant une difficulté relative au fait d'avoir des enfants que ce thème est abordé. En effet, comme l'explique un médecin, aucune aide telle que le DPN ou le DPI ne peut leur être proposée. Il est arrivé à tous les médecins généticiens que nous avons rencontrés de recevoir des personnes qui évoquaient ces deux techniques : dans ce cas, une consultation avec le chef de service leur était proposée. Cette manière d'éviter les questions relatives à la procréation pose des problèmes aux médecins comme aux personnes concernées.

\subsection{Le malaise des médecins}

D'après la grande majorité des entretiens, aborder la question de la reproduction dans le contexte d'un risque de transmission des mutations BRCA1/2 met les médecins « mal à l'aise ». Des 
exemples extraits de leurs consultations leur venaient alors à l'esprit pour expliquer ce malaise : une

patiente exprime combien il est «atroce » de savoir qu'elle peut transmettre la mutation. Une autre explique qu'elle fait un régime garçon. Certains médecins rapportent aussi que leurs patientes élaborent des solutions qu'elles présentent alors en consultation, comme cette femme qui s'est renseignée sur la possibilité de recourir au don d'ovocyte : cela lui permettrait d'une part de ne pas prendre le risque de transmettre la mutation génétique et d'autre part de procéder à une ovariectomie prophylactique, recommandée dans son cas. Un médecin chargé des consultations d'information et de rendu de résultat du test génétique résume l'embarras qu'il peut ressentir lors des consultations qu'il mène avec des femmes et des hommes encore en âge d'avoir des enfants : il se demande si le fait de connaître leur statut génétique ne va pas «les empêcher d'avoir des enfants ». D'après ces médecins, il arrive en effet que les patients demandent quels sont les risques pour leurs enfants, questionnent sur les moyens de les éviter et expliquent enfin qu'ils n'auront sans doute pas d'enfant.

Dans les deux centres où nous avons réalisé notre étude, les femmes âgées de moins de 25 ans devaient rencontrer un psychologue ou un psychiatre rattaché au service avant de réaliser le test génétique. Même quand ce problème n'est pas abordé de front dans leurs consultations, les médecins généticiens savent en effet que cette information peut avoir des conséquences sur leurs choix reproductifs. Un psychologue explique effectivement que sa consultation est sans doute le lieu où certaines questions peuvent être posées et développées. Il en évoque deux: « dois-je finir mon projet parental avant d'avoir mon résultat du test parce que si j'ai le résultat, ce sera beaucoup plus compliqué pour moi d'avoir des enfants ?» «Peut-être devrais-je, si je suis un bon parent, ne pas procréer?». La dimension éthique de ces questions déborde-t-elle le cadre des consultations liées à la réalisation d'un test génétique ? En tous cas, des études montrent qu'elles sont importantes pour les décisions procréatives [4-7].

Dans ce contexte, beaucoup de médecins, qu'ils soient chargés des consultations de conseil génétique ou de la surveillance des femmes à risque de développer un cancer, expriment le souhait qu'une «réflexion formalisée » soit menée à propos du diagnostic anténatal afin que des 
recommandations puissent être publiées. Ils souhaitent en effet pouvoir se référer, pendant leurs consultations, à un cadre et à un discours formalisé.

\subsection{L'impact de l'information génétique sur les choix reproductifs}

Notre enquête suggère également que bien souvent les personnes qui portent une mutation BRCA1/2 ne prévoient pas l'ampleur de l'impact de cette information sur leurs choix reproductifs et ceux de leurs enfants [5]. Les consultations de génétique ont principalement pour fin de mettre en place des mesures de prévention et de surveillance, mais les femmes rapportent par exemple que la prévision d'une ovariectomie les amènent souvent à remettre en question leurs projets d'enfants. Pour certaines d'entre elles, prévoir cette opération crée un sentiment d'urgence difficilement compatible avec le temps de réflexion dont elles ont besoin pour savoir si elles peuvent assumer le risque de transmettre leur mutation génétique.

Les femmes plus âgées expliquent parfois que le résultat du test génétique a eu des conséquences qu'elles ne soupçonnaient pas sur les choix reproductifs de leurs enfants. Ainsi, une femme en cours de traitement pour un second cancer du sein raconte qu'elle a fait le test génétique pour ses enfants âgés d'une trentaine d'années, une femme elle-même mère de deux enfants et un homme en couple sans enfant. Elle est affligée d'une décision qu'elle n'a pas anticipée et dont elle se sent responsable : son fils, qui à son tour a réalisé le test génétique, a décidé qu'il n'aurait pas d'enfants.

\subsection{Le rôle de la famille dans l'accès à l'information génétique}

L'article L1131-1-2 du Code de la santé publique en date du 7 juillet 2011 stipule: «Préalablement à la réalisation d'un examen des caractéristiques génétiques d'une personne, le médecin prescripteur informe celle-ci des risques qu'un silence ferait courir aux membres de sa famille potentiellement concernés si une anomalie génétique grave dont les conséquences sont susceptibles de mesures de prévention, y compris de conseil génétique, ou de soins était 
diagnostiquée ». Lors des consultations de génétique, un moment important consiste donc à

préparer la transmission de l'information génétique dans la famille. Cette tâche n'est pas facile. Parmi les personnes que nous avons rencontrées, certaines ont déploré le manque de communication familiale au sujet de la maladie. De plus, le plus souvent, la démarche de recherche génétique n'est pas considérée comme une démarche familiale, mais personnelle. Ainsi les apparentés en sont-ils informés après coup sans qu'aucun échange à ce sujet n'ait eu lieu.

Dans le contexte du cancer du sein et des ovaires, la transmission de l'information génétique suit une éthique particulière [5]. Le messager considère en effet la situation de chaque apparenté et juge du moment opportun pour lui transmettre l'information. Par exemple, les femmes que nous avons rencontrées préfèrent reporter ce moment si leur fille rencontre des problèmes de couple. Certaines personnes rapportent également avoir attendu que leurs apparentées aient eu tous leurs enfants. Mais cela pose problème eu égard au respect de leur liberté et de leur autonomie reproductive : pour certaines personnes, il était en effet exclu de prendre le risque de transmettre une mutation BRCA1/2 à leurs enfants.

Comme le souligne un médecin, les médecins doivent faire face aujourd'hui à une lourde responsabilité : ils doivent décider d'introduire ou non les informations concernant le diagnostic anténatal dans les consultations relatives à la prise en charge du cancer du sein et des ovaires. Notre enquête suggère qu'ils souhaitent prendre cette responsabilité collégialement et avoir une position claire sur la question. Dans une étude récente [21], des hommes et des femmes porteurs de mutations BRCA1/2 et qui n'avaient pas développé de cancer étaient interrogés sur l'impact des tests génétiques sur leurs projets d'enfants. A supposer que le DPN et le DPI soient autorisés en France pour cette indication, $85,4 \%$ des personnes pensaient que l'information concernant ces pratiques devrait être délivrée systématiquement avec le résultat du test génétique. Notre étude montre cependant l'intérêt d'aborder les questions relatives au projet d'enfant avant la réalisation du test génétique : il s'agit de bien mesurer tous les impacts que cette information pourrait avoir sur les 
choix reproductifs des différents membres de la famille. Elle souligne également l'intérêt d'aborder

ce thème avec la personne chargée de transmettre l'information dans la famille. Enfin, elle suggère que, comme dans le cas du rétinoblastome, les personnes concernées souhaiteraient être informées pour faire les choix qui leur paraissent les meilleurs.

\section{3) Transmission des informations médicales et décision partagée}

Lorsque les couples prennent des décisions procréatives, ils reçoivent un certain nombre d'informations de la part des médecins, ainsi que des avis et éventuellement des conseils ${ }^{4}$. Nos entretiens permettent de mieux comprendre à quelles conditions la transmission de l'information médicale permet une décision libre et autonome.

\subsection{Une décision partagée}

Pour le rétinoblastome et la PAF, les médecins insistent sur leur devoir de transmettre les informations relatives à la prise en charge de la maladie et au diagnostic anténatal aux couples qu'ils rencontrent. L'un d'entre eux souligne qu'inévitablement, en choisissant les informations les plus adaptées à la situation, il oriente leur réflexion. De leur côté, les personnes que nous avons rencontrées n'attendaient pas particulièrement des médecins une information neutre. Elles rapportent que les médecins avec qui elles ont discuté du DPN et du DPI informent, donnent leur avis et des conseils.

Dans ce contexte, ces personnes ont pu prendre la décision qui leur paraissait la meilleure. Comme d'autres, une femme avait par exemple compris que l'option de poursuivre la grossesse après un DPN «n'était pas forcément ... recevable ». Pourtant, la mère d'un enfant atteint de rétinoblastome raconte : «ils m'ont passée par un psychologue, puis j'ai fait le test prénatal ». Elle rit en disant : «ils ne m'ont pas du tout bien diagnostiquée !». Contrairement à ce qui avait été envisagé et discuté avec le psychologue, c'est-à-dire un DPN « en prévision d'avorter si l'enfant était 
atteint », elle a finalement décidé de poursuivre sa grossesse. Malgré une prise en charge médicale

qui lui a semblé parfois contraignante, cette femme a pris, avec son mari, la décision qui leur paraissait la meilleure.

D'après les personnes qui ont envisagé d'avoir recours au DPN ou au DPI pour éviter de transmettre une mutation des gènes $R B 1$ ou $A P C$, les médecins n'imposent pas leur point de vue et respectent leurs choix. Lorsqu'il évoque le moment où la décision de recourir au DPI a été prise, un homme utilise la formule suivante : «le moment où on [lui et son épouse] a pris la décision avec $\mathrm{X}$ [le médecin] ». C'est cette fois une décision partagée qui est évoquée ${ }^{5}$. Dans ce cas, les couples concernés sont bien les auteurs de leurs décisions, et en ce sens sont autonomes. Nos entretiens suggèrent que la compréhension des informations sur lesquelles sont basés les avis médicaux est une des conditions de ce type de décision ${ }^{6}$.

\subsection{La compréhension des avis médicaux}

Les attitudes des personnes face aux avis médicaux peuvent être très différentes. Un homme affirme : «Moi on m'a dit «faut faire ça » donc je suis l'avis des médecins ». Un médecin décrit certains patients au «garde à vous »: ils attendent les ordres et les exécutent, se soumettant à l'autorité avec confiance. Cette attitude hétéronome ne requiert pas la compréhension des informations médicales. En ce qui concerne les décisions partagées, au contraire, nos entretiens suggèrent que les couples cherchent non seulement à comprendre, mais aussi à évaluer la connaissance et l'expérience médicales qui fondent les avis des médecins.

Les personnes que nous avons rencontrées prennent d'autant plus volontiers en compte dans leurs réflexions et leurs décisions les avis des médecins qu'ils leur semblent fondés objectivement. Ils sont particulièrement sensibles au fait que la connaissance soit partagée par différents médecins et que leur expérience dépasse le cadre de leur situation particulière. Dans ce cas en effet, l'avis médical ne dépend pas de l'arbitraire d'un seul médecin. Au contraire, une jeune femme explique

5 On peut trouver dans [22] de beaux exemples de décisions partagées de recourir au DPN.

6 Deux autres conditions sont la prise en compte du projet du couple et de ses besoins spécifiques. Nous ne pouvons les développer dans le cadre de cette publication. 
qu'elle a réalisé un test génétique dans le cadre d'une procédure de procréation médicalement

assistée. Se méfiant des effets de la stimulation hormonale sur le risque de développer des tumeurs au niveau des seins et des ovaires, la gynécologue lui avait dit : « si vous êtes porteuse, on ne fera rien ». Cette jeune femme revient sur le désarroi qu'elle a éprouvé à l'époque et explique qu'elle a alors appelé un autre centre : «je m'étais dit: au pire, s'ils ne veulent pas, j'irai ailleurs ». Le médecin du second centre était d'accord pour la prendre en charge même si elle était porteuse.

Lorsque les conditions de la compréhension des données et de l'expérience médicales ne sont pas réunies, les avis médicaux peuvent être perçus comme extrêmement contraignants. Une femme raconte qu'un an et demi après la fin du traitement contre un cancer du sein, elle est tombée enceinte. Elle rapporte : «on m'a demandé d'avorter». Elle raconte : «j'ai vraiment suivi les prescriptions des médecins sans avoir le sentiment d'avoir eu une explication claire, nette, précise sur le pourquoi du comment, sur quel était, quelle est ou quelles sont les raisons de cet avortement, qu'est-ce qui le motive réellement. Heu... on m'a dit: «non, c'est pas possible, dans votre situation » ». Au moment de l'entretien, et après avoir redemandé des explications aux médecins du centre et à sa gynécologue, elle affirmait avoir encore des doutes sur le bien-fondé de cette décision d'avorter.

\subsection{Les limites de la compréhension des informations médicales}

Les médecins que nous avons rencontrés étaient tous soucieux de la compréhension des informations qu'ils transmettent. Certains expliquaient de quelle manière ils s'en assuraient pendant leurs consultations. D'autres insistaient sur la nécessité d'adresser les couples aux médecins chargés de la prise en charge de la maladie afin qu'ils puissent s'en faire l'idée la plus juste possible. Les informations les plus précises possibles sur le traitement des tumeurs ou les chirurgies prophylactiques sont en effet précieuses pour prendre des décisions procréatives. Malgré tout, l'un des médecins généticiens était fort peu optimiste quant à la qualité de la compréhension de ses patients. Quelques entretiens ont effectivement souligné un manque d'information ou de 
compréhension des données médicales concernant la maladie, sa prise en charge, le risque de

transmission et les moyens de l'éviter. Un psychologue s'interroge également sur la notion de consentement «éclairé » en ce qui concerne la réalisation d'un test génétique. Dans la mesure où les problématiques familiales ne sont pas creusées en consultation, il se demande comment les patients peuvent « maturer » leur décision.

Les personnes que nous avons rencontrées ont également fait l'expérience des limites de la connaissance médicale. Par exemple, un couple explique qu'à l'issue d'un DPI, ils auraient souhaité que soient réimplantés deux embryons pour avoir des jumeaux. Cependant, ils comprennent bien que les médecins ne savent pas si transférer deux embryons confère plus de chances d'avoir un enfant. Finalement, la meilleure solution est trouvée en fonction du projet du couple : «[les médecins] disent «mine de rien, un accouchement prématuré à six mois peut quand même avoir des grosses séquelles », et ils disent «si on a fait tout ça [la FIV et le DPI] pour éviter que votre enfant soit malade et qu'au final vous vous retrouvez avec un gamin anormal parce qu'il est né à six mois de grossesse, vous n'êtes pas plus avancés » ». Le couple a donc choisi de transférer un seul embryon.

Pour certaines personnes, prendre une décision procréative était particulièrement difficile. Elles expliquent alors qu'il est important d'être l'auteur de la décision : c'est à cette condition que le choix peut être assumé et la situation qui en découle supportée. Cependant, être auteur de la décision ne signifie pas nécessairement la prendre seul. Dans ces circonstances, il est en effet important de comprendre les avis médicaux et de se référer avec les médecins à un projet. Enfin, la connaissance médicale et la connaissance de soi-même (notamment en lien avec l'histoire familiale) sont nécessairement limitées puisque, de manière différentes, leurs objets sont inépuisables [23]. Un objectif du conseil génétique pourrait donc être de transmettre aux couples des informations dont la pertinence serait établie en fonction du processus complexe (dont la dimension médicale n'est qu'une dimension) de prise de décision autonome. 


\section{Conclusion}

En raison du petit nombre d'entretiens, les résultats présentés ici ne peuvent avoir qu'une valeur exploratoire. De plus, nous n'avons rencontré aucune personne âgée de moins de 25 ans au moment de faire un test génétique BRCA1/2. En ce qui concerne le rétinoblastome et la PAF, nous n'avons rencontré aucun couple dont la demande de DPN ou de DPI aurait été refusée. Néanmoins, notre enquête engage plusieurs pistes de réflexion sur l'influence des modalités de transmission de l'information médicale sur les réflexions et les décisions des personnes qui envisagent d'avoir un enfant alors qu'elles risquent de lui transmettre une anomalie génétique.

Les liens entre l'autonomie des personnes et leur liberté de décision d'une part et l'accès à l'information et la compréhension des données médicales d'autre part ont été soulignés et discutés [23-26]. La comparaison des entretiens avec des personnes porteuses d'une mutation des gènes $R B 1$, BRCA1 ou BRCA2 souligne l'importance des modalités d'accès à l'information génétique. Selon la manière dont la prise en charge des malades et de leur famille est organisée, et selon la place faite ou non en consultation de génétique aux discussions sur le projet d'enfant, les couples disposent plus ou moins facilement des informations utiles pour prendre les décisions procréatives qu'ils estiment les meilleures.

L'impact des choix des médecins concernant les informations à transmettre et les modalités de prise en charge est donc important. Même lorsque la famille est le principal vecteur de la transmission de l'information génétique, ces choix peuvent favoriser ou non l'autonomie des personnes concernées par la transmission d'une anomalie génétique. Dans le contexte du cancer du sein et des ovaires, nos résultats soulignent en effet l'intérêt qu'il y aurait à aborder les questions relatives à la procréation bien en amont de la réalisation du test génétique avec la personne chargée de la transmission de l'information dans la famille.

Enfin, notre enquête appelle à mettre en perspective l'importance des données médicales pour les décisions procréatives. En effet, les médecins envisagent les réflexions relatives au projet 
d'enfant sous l'angle des solutions qu'ils peuvent proposer pour éviter le risque de transmettre une anomalie génétique. En outre, les décisions reproductives prises par le couple et le médecin reposent sur un partage de la connaissance et de l'expérience médicales. Enfin, dans son avis n 107 [12], le CCNE explique que le critère de la gravité de la maladie doit guider les CPDPN lorsqu'ils examinent un dossier de demande de diagnostic anténatal. Mais pour les couples, le questionnement est plus vaste : il fait aussi appel à des considérations éthiques, notamment sur le fait d'être un bon parent. Il conviendrait donc de s'interroger : quelle place donner à ces considérations, notamment dans les décisions de recourir au diagnostic prénatal et préimplantatoire?

\section{Remerciements}

Je remercie XXX, avec qui j'ai mené cette enquête, les médecins qui nous ont accueillis dans leurs centres, et toutes les personnes qui ont accepté de nous rencontrer.

\section{Aucun conflit d'intérêt}




\section{Références bibliographiques}

1. Lammens C., Bleiker E., Aaronson N., Vriends A. Attitude towards preimplantation genetic diagnosis for hereditary cancer. Familial Cancer $2009 ; 8$ : 457-64

2. Kastrinos K., Stoffel E.M., Balmaña J. Attitudes towards prenatal genetic testing in patients with familial adenomatous polyposis. Am J Gastroenterol 2007 ; 102 : 1-7

3.

4. Quinn G.P., Vadaparampil S.T., King L.M., Miree C.A., Friedman S. Conflict between values and technology : perceptions of preimplantation genetic diagnosis among women at increased risk for hereditary breast and ovarian cancer. 2009 Familial Cancer ; $8: 441-9$

5.

6. Hallowell N. Arden-Jones A. Eeles R. Foster C. Lucassen A. Moynihan C. et al. Guilt, blame and responsibility : men's understanding of their role in the transmission of BRCA1/2 mutations within their family. Sociol Health Illn 2006 ; 28: 969-88

7. Rowley E. On doing «being ordinary»: women's account of BRCA testing and maternal responsibility. New Genet Soc $2008 ; 26: 241-50$

8. Conseil de l'Europe, Protocole additionnel à la Convention sur les Droits de l'Homme et la biomédecine relatif aux tests génétiques à des fins médicales, Strasbourg, 27 novembre 2008 http://conventions.coe.int/Treaty/fr/Treaties/html/203.htm.

Rapport explicatif : http://conventions.coe.int/Treaty/FR/Reports/Html/203.htm

9. Paul D. The politics of heredity. Essays on eugenics, biomedicine, and the nature-nurture debate, Albany : State University of New York Press, 1998, 219 pages

10. Hodgson J Spriggs M. A practical account of autonomy : why genetic counseling is especially well suited to the facilitation of informed autonomous decision making. J Genet Couns $2005 ; 14$ : 89-97

11. Skirton H, Goldsmith L, Jackson L, Tibben A. Quality in genetic counselling for presymptomatic testing - clinical guidelines for practice across the range of genetic conditions. Eur 
J Hum Genet 2012 Aug 15, à paraître

12. Comité Consultatif National d'Ethique pour les Sciences de la Vie et de la Santé, Avis n 107 sur les problèmes éthiques liés aux diagnostics anténatals : le diagnostic prénatal (DPN) et le diagnostic préimplantatoire (DPI), 15 octobre 2009 http://www.ccne-ethique.fr/docs/Avis

13. Code de la santé publique, articles L. 2213-1 et L. 2131-4 http://www.legifrance.gouv.fr

14. Clarke A. Is non-directive counseling possible ? Lancet $1991 ; 338: 1284-90$

15. Oduncu FC. The rôle of non-directiveness in genetic counseling. Med health Care and Philos $2002 ; 5: 53-63$

16. Diagnostic prénatal, interruption médicale de grossesse, diagnostic préimplantatoire et formes héréditaires de cancers. Rapport rédigé à la demande de l'Agence de la Biomédecine et de l'Institut National du Cancer. Avril 2008. http://www.agence-biomedecine.fr/professionnels/rapports-etetudes-realises-autour-de-l-activite-de-dpi.html

17. http://www.agence-biomedecine.fr/annexes/bilan2011/donnees/diagprenat/01diag_prenat/synthese.htm

18. http://www.agence-biomedecine.fr/annexes/bilan2011/donnees/diag-prenat/03preimpl/synthese.htm

19. Levy-Lahad E. Friedman E. Cancer risk among BRCA1 and BRCA2 mutation carriers. Br J Cancer 2005 ; 96: 11-5

20. Fortuny D. Balmaña J. Graña B. Torres A. Ramón y Cajal T., Darder E. et al. Opinion about reproductive decision making among individuals undergoing BRCA1/2 genetic testing in a multicentre Spanish cohort. Human Reprod 2009 ; 24 : 1000-6

21. Julian-Reynier C. Fabre R. Coupier I. Stoppa-Lyonnet D. Lasset C. Caron O. et al. BRCA1/2 carriers : their childbearing plans and theoretical intentions about having Preimplantation Genetic Diagnosis and Prenatal Diagnosis. Genet Med 2012 ; 14 : 527-34

22. Gaille M. Viot G. Prenatal Diagnosis as a tool and support for eugenics : myth or reality in contemporary French society ? Med Health Care and Philos 2012. Published online : 20 July 2012 
23. Marzano M. Je consens donc je suis. Ethique de l'autonomie, Paris : PUF, 2006, 261 pages

24. Hottois G. Essais de philosophie bioéthique et biopolitique, Paris : Vrin, 1999, 189 pages

25. Harris J. Keywood K. Ignorance, information and autonomy. Theoretical Medicine and Bioethics $2001 ; 22: 415-36$

26. Häyry M. Takala T. Genetic information, rights, and autonomy, Theoretical Medicine and Bioethics, $2001 ; 22: 403-14$ 
Tableau 1

\begin{tabular}{|c|c|c|c|}
\hline & RB1 & APC & BRCA1/2 \\
\hline Sexe & $\begin{array}{l}7 \text { hommes } \\
3 \text { femmes }\end{array}$ & 2 hommes & $\begin{array}{l}19 \text { femmes } \\
2 \text { hommes }\end{array}$ \\
\hline \multicolumn{4}{|l|}{ Age } \\
\hline $25-29$ & & 1 & \\
\hline 30-39 & 8 & 1 & 11 \\
\hline $40-49$ & 2 & & 5 \\
\hline $50-59$ & & & 4 \\
\hline 60 et plus & & & 1 \\
\hline \multicolumn{4}{|l|}{ État civil } \\
\hline Marié / en couple & 10 & 2 & 17 \\
\hline Divorcé & & & 3 \\
\hline Célibataire & & & 1 \\
\hline \multicolumn{4}{|l|}{ Nombre d'enfants } \\
\hline Aucun & 2 & 1 & 5 \\
\hline 1 & 5 & 1 & $\begin{array}{l}6 \text { (dont } 2 \text { femmes } \\
\text { enceintes du second } \\
\text { enfant) }\end{array}$ \\
\hline 2 & 3 & & 8 \\
\hline 3 & & & 1 \\
\hline Recours au DPN & $\begin{array}{l}4 \\
1 \text { IMG } \\
\text { Naissance de } 2 \text { enfants } \\
\text { non porteurs et d'un } \\
\text { enfant porteur. }\end{array}$ & & \\
\hline Recours au DPI & $\begin{array}{l}1 \text { (5 essais, pas de } \\
\text { naissance) } \\
\text { deux dossiers en cours } \\
\text { d'examen }\end{array}$ & 1 (1 naissance) & $\begin{array}{l}2 \text { ( } 2 \text { naissances) } \\
\text { Les DPI ont été réalisés } \\
\text { à l'étranger }\end{array}$ \\
\hline
\end{tabular}


Tableau 1

Profil démographique des personnes rencontrées

\begin{tabular}{|c|c|c|c|}
\hline & RB1 & APC & BRCA1/2 \\
\hline Sexe & $\begin{array}{l}7 \text { hommes } \\
3 \text { femmes }\end{array}$ & 2 hommes & $\begin{array}{l}19 \text { femmes } \\
2 \text { hommes }\end{array}$ \\
\hline \multicolumn{4}{|l|}{ Age } \\
\hline $25-29$ & & 1 & \\
\hline 30-39 & 8 & 1 & 11 \\
\hline $40-49$ & 2 & & 5 \\
\hline $50-59$ & & & 4 \\
\hline 60 et plus & & & 1 \\
\hline \multicolumn{4}{|l|}{ État civil } \\
\hline Marié / en couple & 10 & 2 & 17 \\
\hline Divorcé & & & 3 \\
\hline Célibataire & & & 1 \\
\hline \multicolumn{4}{|l|}{ Nombre d'enfants } \\
\hline Aucun & 2 & 1 & 5 \\
\hline 1 & 5 & 1 & $\begin{array}{l}6 \text { (dont } 2 \text { femmes } \\
\text { enceintes du second } \\
\text { enfant) }\end{array}$ \\
\hline 2 & 3 & & 8 \\
\hline 3 & & & 1 \\
\hline Recours au DPN & $\begin{array}{l}4 \\
1 \text { IMG } \\
\text { Naissance de } 2 \text { enfants } \\
\text { non porteurs et d'un } \\
\text { enfant porteur. }\end{array}$ & & \\
\hline Recours au DPI & $\begin{array}{l}1 \text { (5 essais, pas de } \\
\text { naissance) } \\
\text { deux dossiers en cours } \\
\text { d'examen }\end{array}$ & 1 (1 naissance) & $\begin{array}{l}2 \text { ( } 2 \text { naissances) } \\
\text { Les DPI ont été réalisés } \\
\text { à l'étranger }\end{array}$ \\
\hline
\end{tabular}

